\title{
Deep vein thrombosis and pulmonary embolism in pediatric COVID-19
}

\author{
Loukia Ioannidou $^{1}$, Athina Dettoraki ${ }^{1}$, Maria Noni ${ }^{2}$, Dimitra Koukou ${ }^{1}$, Aiketarini \\ Michalopoulou $^{1}$, Zoey Kapsimalli ${ }^{1}$, Evanthia Botsa ${ }^{3}$, Athanasios Michos ${ }^{4}$, Vana Spoulou ${ }^{2}$, \\ Helen Pergantou ${ }^{1}$, and Christina Kanaka-Gantenbein ${ }^{3}$ \\ ${ }^{1}$ Agia Sofia Children's Hospital \\ ${ }^{2}$ National and Kapodistrian University of Athens \\ ${ }^{3}$ National and Kapodistrian University of Athens - Faculty of Medicine \\ ${ }^{4}$ University of Athens
}

September 26, 2021

\begin{abstract}
Thrombotic complications of SARS-CoV-2 have been increasingly recognized as an important component of COVID-19 in adults; however, they have been less evident in children. We report a case of a teenager with positive SARS-CoV-2 RT-PCR and underlying prothrombotic risk factors, including aromatase inhibitor therapy, who developed deep vein thrombosis resulting in pulmonary embolism. Laboratory tests revealed deranged coagulation parameters (high D-dimers and Factor VIII and low antithrombin). The patient required intensive care and was managed with anticoagulants, dexamethasone and antithrombin concentrate. Clinical condition and hemostatic profile gradually improved. A review of the available literature for similar cases is presented.
\end{abstract}

\section{Introduction}

Coronavirus disease 2019 (COVID-19), caused by severe acute respiratory syndrome coronavirus 2 (SARS$\mathrm{CoV}-2$ ), has various extra-pulmonary manifestations, including venous thromboembolism (VTE) ${ }^{1}$. Pediatric recommendations for hospital-associated VTE in COVID-19 have been provided ${ }^{2,3}$, though data regarding VTE risk in non-hospitalized children are limited. We describe the case of an adolescent with a positive SARS-CoV-2 reverse transcription polymerase chain reaction (RT -PCR) and underlying prothrombotic risk factors who developed deep vein thrombosis (DVT), resulting in pulmonary embolism (PE).

\section{Case Description}

A 15-year-old male was referred to our emergency department with a four-day history of gradually progressive pain and swelling of the right lower limb, in the absence of any recent history of injury. A magnetic resonance imaging (MRI) of the hip, undertaken previously in outpatient setting following orthopedic referral, demonstrated a thrombus in the external iliac vein(Figs. 1A and 1B). The patient also reported gastrointestinal symptoms two weeks earlier, i.e., diarrhea and vomiting, along with low-grade fever of $37.3 \mathrm{oC}$, lasting for two days. With regards to his medical history, he was under anastrozole, an aromatase inhibitor (AI), over the last two years, as prescribed by his physician for short stature.

The adolescent was overweight with body mass index (BMI) of $25.2 \mathrm{~kg} / \mathrm{m}^{2}$. On admission, he had body temperature of $38.9 \mathrm{oC}$ and exhibited pain in the right hip with limited mobility of the joint and edema of the right knee. Triplex ultrasound of the right lower extremity depicted an extended DVT, from the external iliac vein down to the great saphenous and popliteal vein. Further laboratory investigations were 
undertaken, including SARS-CoV-2 RT-PCR on nasopharyngeal sample. Initial management consisted of combined antibiotic therapy with intravenous (IV) ceftriaxone and teicoplanin and anticoagulation treatment with subcutaneous low molecular weight heparin (LMWH) i.e., tinzaparin in a therapeutic dose of $175 \mathrm{IU} /$ $\mathrm{kg}$ body weight once daily.

Within the first hour, he developed hypotension with low diastolic blood pressure $(25 \mathrm{mmHg})$, not responding to IV fluids, tachycardia (125 beats/min) and reduced urine output. Saturation of oxygen dropped to $94 \%$, few hours later. Computed tomography pulmonary angiogram (CTPA) demonstrated embolus in the left pulmonary artery. (Figs. 1C and 1D), whereas the SARS-CoV-2 RT-PCR revealed high viral load. ${ }^{4}$

The patient was urgently transferred to the COVID-19 pediatric intensive care unit where IV dexamethasone $0.15 \mathrm{mg} / \mathrm{kg}(\max 6 \mathrm{mg} /$ day) was added to his treatment plan. Oxygen supply was maintained to $2-3 \mathrm{~L} / \mathrm{min}$ without further need of intubation or mechanical ventilation. Subcutaneous tinzaparin was continued and a single dose of IV antithrombin concentrate was administered due to low levels, resulting in immediate response to $125 \%$. Systematic thrombolysis was not required. High temperature subsided 24 hours later, oxygen was weaned off and antibiotic treatment was discontinued following negative blood cultures.

The patient stepped down to the COVID-19 pediatric ward after 72 hours where he gradually improved. A total of ten days of IV dexamethasone was completed and subcutaneous tinzaparin was substituted by oral warfarin, after completion of three weeks. Anastrozole was discontinued. He was discharged34 days after admission under warfarin $5 \mathrm{mg}$ once daily. Hemostatic parameters and SARS-CoV-2 RT-PCR were regularly evaluated during hospital stay and post discharge, as summarized in Table 1. Genetic testing for hereditary thrombophilia has been scheduled after the acute phase of thrombosis.

\section{Discussion}

Only few patients under 18 years with COVID-19 have been reported with $\mathrm{PE}^{5-7}$. The vast majority of literature data refers to critically ill adults ${ }^{8-10}$, where local pulmonary thrombotic microangiopathy appears to be the underlying pathophysiological mechanism ${ }^{11}$. In contrast, our patient had mild COVID-19 prior to manifestation of VTE, without hyperinflammatory state, and PE was secondary to embolization from the lower limb.

Puberty and age above 12 years increase the thrombotic risk in children ${ }^{2}$. Nevertheless, the adolescent had additional prothrombotic risk factors. Aromatase inhibitor (AI) therapies prevent the conversion of androgens to estrogens and are mainly used in estrogen receptor-positive breast cancer. In children with short stature, they are supposed to allow for greater height potential by delaying the epiphysial maturation, given the fact that the ultimate fusion of the growth plates in both males and females is estrogen-dependent ${ }^{12}$. Thromboembolism is an adverse effect of AI in female breast cancer patients and women receiving anastrozole are found to have greater risk for VTE compared to untreated, healthy women ${ }^{13}$. Such adverse effects have been poorly investigated in children. Therefore, the inhibition of the estrogens production through the inhibition of the aromatase may lead to increased testosterone concentration. In turn, the increased testosterone levels in pubertal boys could lead to erythrocytosis and thrombotic events, but further investigation is needed in this context ${ }^{14}$. Moreover, in our patient, the reported pre-existing symptoms from gastrointestinal system could induce hypercoagulability in view of dehydration and increased blood viscosity, as described in adults with COVID-19 ${ }^{15}$. The patient also reported excessive screen time due to his tele-education over the last weeks and the resulting immobilization, i.e. being sedentary for many hours, could constitute an additional risk factor through circulatory stasis, as described under the term of e-thrombosis ${ }^{16}$.

COVID-19 as well induces per se a hypercoagulable state ${ }^{17}$. The suggested mechanism ${ }^{18,19}$ is that the disease leads to an immunothrombosis response through the interplay between inflammatory and coagulation pathways, resulting in cytokine storm, neutrophil and complement activation which propagate a pro-agulant state. Furthermore, the virus itself provokes a direct endothelial injury activating the coagulation cascade. The vascular endothelium is a key target-organ of SARS-Cov- $2^{20}$ this endotheliitis ${ }^{21}$ was evident in our case, as high levels of Factor VIII (FVIII) were persistent. FVIII is a blood-clotting protein associated with inflammation but also direct endothelial damage ${ }^{22}$ and it has been suggested as a predictive coagulation 
biomarker in COVID-19, as reported in a cohort study of adult patients, where high levels at admission were linked to early-onset $\mathrm{VTE}^{23}$.

Coagulopathy in COVID-19 has further laboratory features, overlapping with other coagulopathies but also differing ${ }^{24,25}$. Similarly, our patient presented high fibrinogen, which normalized soon after the initiation of anticoagulation, and significantly increased levels of D-dimers, which gradually decreased but remained above normal limits even after six weeks. Such elevated D-dimers e.g., $>5$ times the upper limit of normal values, are suggested as a marker for introducing thromboprophylaxis in children hospitalized with COVID19, independently of clinical risk factors for $\mathrm{VTE}^{2}$. Moreover, prothrombin time (PT) was mildly prolonged, with subsequently low Factor VII, whereas activated partial thromboplastin time (aPTT) and platelets count were, unlike in disseminated intravascular coagulation, normal.

The patient, additionally, demonstrated persistent low levels of antithrombin even after supplementation. Interestingly, his mother -who had also a positive SARS-CoV2 RT-PCR but remained asymptomatic- was found with low antithrombin (59\%). During her reevaluation one month later (with negative RT-PCR), levels were almost restored (78\%). These findings support the correlation between the virus and acquired antithrombin deficiency, as seen in studies showing low antithrombin in a high proportion of patients with COVID-1926. This should be taken into consideration when introducing anticoagulation to patients with COVID-19; antithrombin deficiency induces heparin resistance and higher heparin doses or antithrombin concentrateto correct values $<70 \%$, like in our case, might be needed ${ }^{27,28}$.

In conclusion, our case underlines the fact that pediatric patients with SARS-CoV-2 infection, even the non - hospitalized, could develop serious VTE in the co-existence of underlying prothrombotic risk factors and COVID-19 associated coagulopathy. Clinical suspicion should be high in specific age groups like adolescents, who may receive therapies like AI or are more sedentary during the pandemic. Therefore, there is a need for further recommendations regarding VTE risk assessment, hemostatic monitoring and application of anticoagulation in children and adolescents with COVID-19.

\section{Acknowledgments}

The authors would like to acknowledge the Pediatric Intensive Care Unit of "P \& A Kyriakou" Children's Hospital for the acute management of our patient, all the physicians involved in our patient's care, Dr Irene Pantou, radiologist in Radiology Department of Eginition Hospital, for the assistance in the radiological findings

\section{References}

1. Gupta A, Madhavan MV, Sehgal K, Nair N, Mahajan S, Sehrawat TS, Bikdeli B, Ahluwalia N, Ausiello JC, Wan EY, Freedberg DE et al. Extrapulmonary manifestations of COVID-19. Nat Med. 2020;26:1017-1032.

2. Goldenberg NA, Sochet A, Albisetti M, Biss T, Bonduel M, Jaffray J, MacLaren G, Monagle P, O'Brien S, Raffini L, et al.; Pediatric/Neonatal Hemostasis and Thrombosis Subcommittee of the ISTH SSC. Consensus-based clinical recommendations and research priorities for anticoagulant thromboprophylaxis in children hospitalized for COVID-19-related illness. J Thromb Haemost. 2020;18:3099-3105.

3. Loi M, Branchford B, Kim J, Self C, Nuss R. COVID-19 anticoagulation recommendations in children. Pediatr Blood Cancer. 2020;67:e28485.

4. Mathuria JP, Yadav R, Rajkumar. Laboratory diagnosis of SARS-CoV-2 - A review of current methods. J Infect Public Health. 2020;13(7):901-905.

5. Kotula JJ, Balakumar N, Khan D, Patel B. Bilateral pulmonary emboli in a teenager with positive SARS-CoV-2 antibody. Pediatr Pulmonol. 2021;56:271-273.

6. Martinelli I, Ferrazzi E, Ciavarella A, Erra R, Iurlaro E, Ossola M, Lombardi A, Blasi F, Mosca F, Peyvandi F. Pulmonary embolism in a young pregnant woman with COVID-19. Thromb Res. 2020;191:36-37

7. Hussain Z, Wangmo R, Gonbo S. Deep Vein Thrombosis After Trivial Blunt Trauma at High Altitude in a SARS-CoV-2 Positive Child: Complication of the Hypercoagulable State. Indian Pediatr. 
2020;57:1182-1183

8. Klok FA, Kruip MJHA, van der Meer NJM, Arbous MS, Gommers DAMPJ, Kant KM, Kaptein FHJ, van Paassen J, Stals MAM, Huisman MV et al. Incidence of thrombotic complications in critically ill ICU patients with COVID-19. Thromb Res. 2020;191:145-147.

9. Iba T, Levy JH, Levi M, Connors JM, Thachil J. Coagulopathy of Coronavirus Disease 2019. Crit Care Med. 2020;48:1358-1364..

10. Middeldorp S, Coppens M, van Haaps TF, Foppen M, Vlaar AP, Müller MCA, Bouman CCS, Beenen LFM, Kootte RS, Heijmans J et al. Incidence of venous thromboembolism in hospitalized patients with COVID-19. J Thromb Haemost. 2020;18:1995-2002.

11. Thachil J, Srivastava A. SARS-2 Coronavirus-Associated Hemostatic Lung Abnormality in COVID-19: Is It Pulmonary Thrombosis or Pulmonary Embolism? Semin Thromb Hemost. 2020;46:777-780.

12. Wit JM, Hero M, Nunez SB. Aromatase inhibitors in pediatrics. Nat Rev Endocrinol. 2011;8:135-47.

13. Baum M, Buzdar A, Cuzick J, Forbes J, Houghton J, Howell A, Sahmoud T; ATAC (Arimidex, Tamoxifen Alone or in Combination) Trialists' Group. Anastrozole alone or in combination with tamoxifen versus tamoxifen alone for adjuvant treatment of postmenopausal women with early-stage breast cancer: results of the ATAC (Arimidex, Tamoxifen Alone or in Combination) trial efficacy and safety update analyses. Cancer. 2003;98:1802-10.

14. Diaz-Thomas A, Shulman D. Use of aromatase inhibitors in children and adolescents: what's new? Curr Opin Pediatr. 2010;22:501-7.

15. Zhou B, She J, Wang Y, Ma X. Venous thrombosis and arteriosclerosis obliterans of lower extremities in a very severe patient with 2019 novel coronavirus disease: a case report. J Thromb Thrombolysis. 2020;50:229-232.

16. Franch-Llasat D, Mayor-Vázquez E, Pedregosa-Díaz J, Herrero-Redondo M, Ortin-Font X, RocheCampo F. e-Thrombosis in the COVID-19 era: collateral effects of confinement. Med Intensiva (Engl Ed). 2021;45:122-124.

17. Abou-Ismail MY, Diamond A, Kapoor S, Arafah Y, Nayak L. The hypercoagulable state in COVID-19: Incidence, pathophysiology, and management. Thromb Res. 2020 Oct;194:101-115.

18. Colling ME, Kanthi Y. COVID-19-associated coagulopathy: An exploration of mechanisms. Vasc Med. 2020;25:471-478

19. Jayarangaiah A, Kariyanna PT, Chen X, Jayarangaiah A, Kumar A. COVID-19-Associated Coagulopathy: An Exacerbated Immunothrombosis Response. Clin Appl Thromb Hemost. 2020;26:1076029620943293.

20. Pons S, Fodil S, Azoulay E, Zafrani L. The vascular endothelium: the cornerstone of organ dysfunction in severe SARS-CoV-2 infection. Crit Care. 2020;24:353

21. Varga Z, Flammer AJ, Steiger P, Haberecker M, Andermatt R, Zinkernagel AS, Mehra MR, Schuepbach RA, Ruschitzka F, Moch H. Endothelial cell infection and endotheliitis in COVID-19. Lancet. 2020;395:1417-1418.

22. Müller MM, Griesmacher A. Markers of endothelial dysfunction. Clin Chem Lab Med. 2000;38:77-85.

23. Rauch A, Labreuche J, Lassalle F, Goutay J, Caplan M, Charbonnier L, Rohn A, Jeanpierre E, Dupont A, Duhamel A et al. Coagulation biomarkers are independent predictors of increased oxygen requirements in COVID-19. J Thromb Haemost. 2020;18:2942-2953

24. Levi M, Iba T. COVID-19 coagulopathy: is it disseminated intravascular coagulation? Intern Emerg Med. 2021;16:309-312.

25. Iba T, Levy JH, Connors JM, Warkentin TE, Thachil J, Levi M. The unique characteristics of COVID19 coagulopathy. Crit Care. 2020;24:360.

26. Gazzaruso C, Paolozzi E, Valenti C, Brocchetta M, Naldani D, Grignani C, Salvucci F, Marino F, Coppola A, Gallotti P. Association between antithrombin and mortality in patients with COVID-19. A possible link with obesity. Nutr Metab Cardiovasc Dis. 2020;30:1914-1919.

27. Maldonado E, Tao D, Mackey K. Antithrombotic Therapies in COVID-19 Disease: a Systematic Review. J Gen Intern Med. 2020;35:2698-2706.

28. Lippi G, Henry BM, Sanchis-Gomar F. Plasma Antithrombin Values Are Significantly Decreased in Co- 
ronavirus Disease 2019 (COVID-19) Patients with Severe Illness. Semin Thromb Hemost. 2021;47:460462.

\section{FIGURE 1}

Imaging confirmation of VTE. Coronal (A) and axial (B) fat-suppressed T2-weighted images of MRI scan of right hip joint, demonstrating a hyperintense clot in the right external iliac vein, and coronal (C) and axial (D) views of lung CTPA scan revealing a large pulmonary embolus of approximately $3 \mathrm{~cm}$ longitudinal diameter in left pulmonary artery's branch which supplies the left lower lobe.

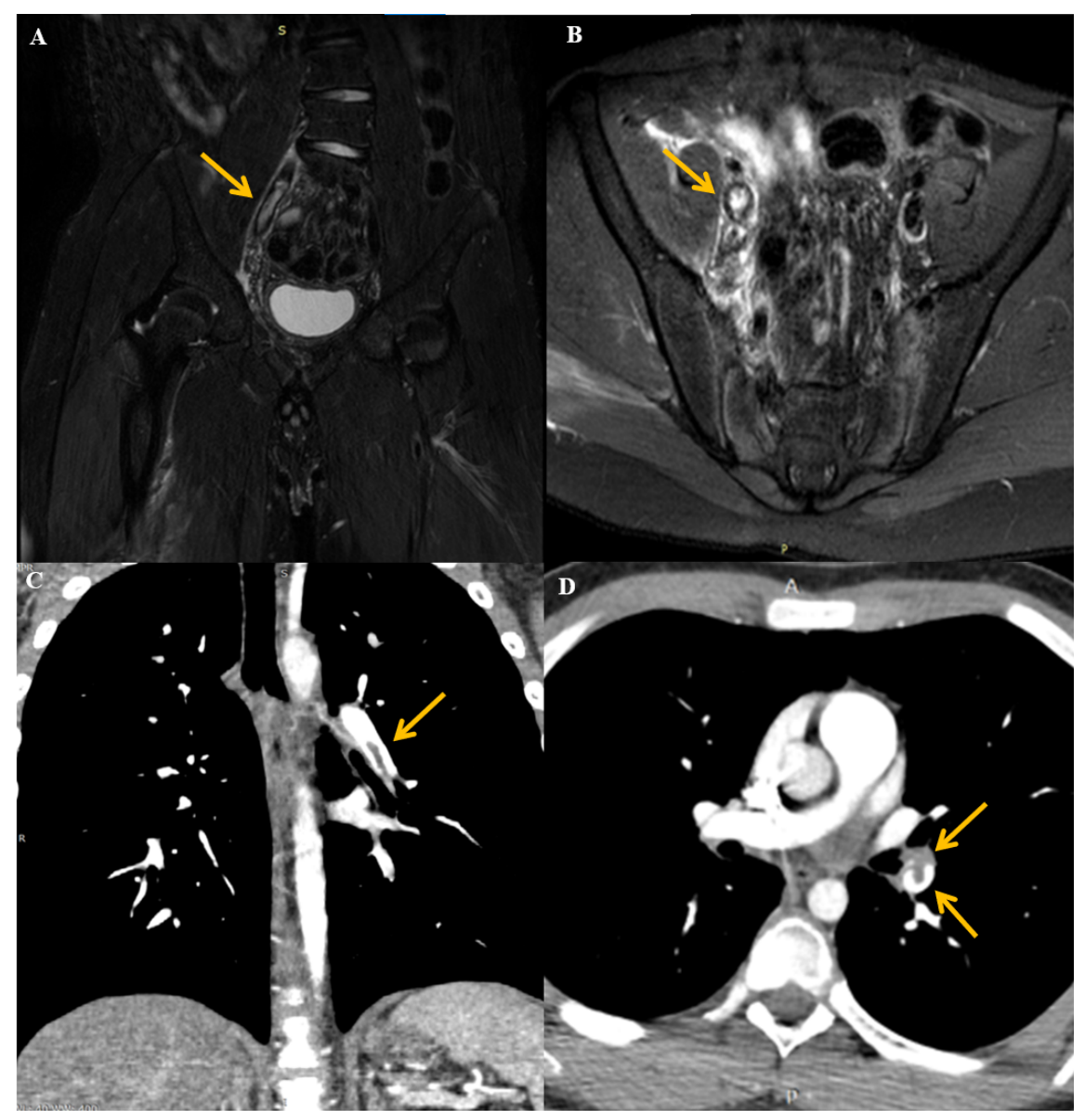

\section{Hosted file}

TABLE 1 Changes in laboratory parameters.docx available at https://authorea.com/users/437900/ articles/539238-deep-vein-thrombosis-and-pulmonary-embolism-in-pediatric-covid-19 